\title{
Evolution of Statistic Moments of 2D-Distributions of Biological Liquid Crystal Net Mueller Matrix Elements in the Process of Their Birefringent Structure Changes
}

\author{
A. G. Ushenko, ${ }^{1}$ I. Z. Misevich, ${ }^{1}$ V. Istratiy, ${ }^{1}$ I. Bachyns'ka, ${ }^{2}$ A. P. Peresunko, ${ }^{2}$ \\ Omar Kamal Numan, ${ }^{2}$ and T. G. Moiysuk ${ }^{2}$ \\ ${ }^{1}$ Optics and Spectroscopy Department, Chernivtsi National University, 2 Kotsyubinsky Street, Chernivtsi 58012, Ukraine \\ ${ }^{2}$ Chernivtsi Medical University, 2 Theatral Square, Chernivtsi 58002, Ukraine
}

Correspondence should be addressed to A. G. Ushenko, yuriyu@gmail.com

Received 3 December 2009; Accepted 29 March 2010

Academic Editor: Oleg V. Angelsky

Copyright (๑) 2010 A. G. Ushenko et al. This is an open access article distributed under the Creative Commons Attribution License, which permits unrestricted use, distribution, and reproduction in any medium, provided the original work is properly cited.

This research is aimed to investigate the reliability of Mueller-matrix differentiation of birefringence change of optically thick layers of biological liquid crystals at the early stages of the change in their physiological state. This is performed by measuring the set of skewness and kurtosis values of Mueller matrix image of the phase element $\mathrm{M}_{44}$ in various points of the object under investigation.

\section{Introduction}

Among many methods of optical diagnostics of organic phase-inhomogeneous object a new technique-laser polarimetry [1] - has been formulated within recent 10 years. It enables to obtain information about optical anisotropy [2-5] of biological tissues (BT) in the form of coordinate distributions of BT Mueller matrix elements, azimuths, and ellipticities of their object field polarization.

To analyze this polarimetric information the following model approach was elaborated [1, 2, 6-13]:

(i) all the variety of human BT can be represented as four main types-connective, muscle, epithelial, and nerve tissues;

(ii) morphological structure of any BT type is regarded as a 2-component amorphous-crystalline structure;

(iii) the crystalline component or extracellular matrix is an architectonic net consisting of coaxial cylindrical protein (collagen, myosin, elastin, etc.) fibrils;

(iv) optically, the protein fibrils possess the properties of uniaxial birefringent crystals;

(v) interaction of laser radiation with the BT layer is considered in the single scattering approximation, when the attenuation factor corresponds to $\tau \leq 0,1$.
Specifically, the above mentioned model was used for finding and substantiating the interconnections between the ensemble of statistic moments of the 1st-4th orders that characterize the orientation-phase structure (distribution of optical axes and phase shifts of protein fibrils networks directions) of birefringent $\mathrm{BT}$ architectonics and that of $2 \mathrm{D}$ distributions of the elements of the corresponding Mueller matrix $[6,10]$. It was determined $[8,11]$ that the $3 \mathrm{rd}$ and the 4 th statistic moments of coordinate distributions of "phase" matrix elements $\left(z_{24}, z_{34}, z_{44}\right)$ are the most sensitive to the change (dystrophic and oncological processes) of optical anisotropy of protein crystals. These statistic moments characterize the BT extracellular matrix birefringence. On this basis the criteria of early diagnostics of muscle dystrophy, precancer states of connective tissue, collagenosis, and so forth were determined.

However, such techniques do not take into account the coordinate heterogeneity of orientation-phase structure of protein crystals nets of the BT layer as well as the order of scattering in its depth. Thus it is important to investigate the distribution of statistic moments of the 1st-4th orders that characterize the 2D elements of Mueller matrix not only in the section of the probing laser beam but also within the whole BT layer of various optical thickness and physiological state. 


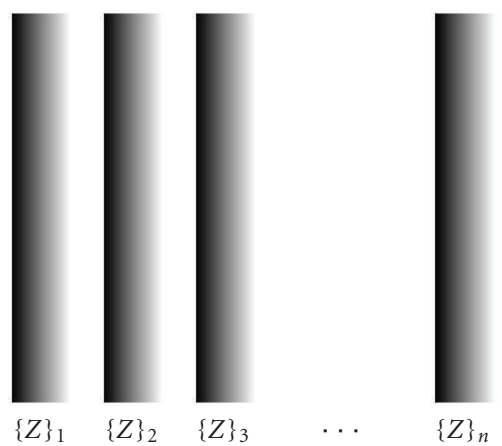

FIGURE 1: On the analysis of modeling polarization properties of anisotropic component of optically thick biological tissue.

For this we shall consider the potentiality of matrix modeling of polarization properties of optically thick BT layer.

\section{Mueller Matrix Modeling of the Properties of Optically Thick Biological Tissue}

Let us represent the layer of such a biological object as the set of successively located optical thin partial layers (Figure 1).

Polarization properties of birefringent nets of every other BT layer are described by the Mueller matrix $\{Z\}_{j}$ representing a superposition of matrix operators of optically separate coaxial protein fibrils $\{z\}_{j}[1]$ :

$$
\{z\}_{j}=\left\|\begin{array}{cccc}
1 & 0 & 0 & 0 \\
0 & z_{22} & z_{23} & z_{24} \\
0 & z_{32} & z_{33} & z_{34} \\
0 & z_{42} & z_{43} & z_{44}
\end{array}\right\|,
$$

where

$$
z_{i k}(\rho, \delta)=\left\{\begin{array}{l}
z_{22}=\cos ^{2} 2 \rho+\sin ^{2} 2 \rho \cos \delta, \\
z_{23,32}=\cos 2 \rho \sin 2 \rho(1-\cos \delta), \\
z_{33}=\sin ^{2} 2 \rho+\cos ^{2} 2 \rho \cos \delta \\
z_{34,43}= \pm \cos 2 \rho \sin \delta \\
z_{24,42}= \pm \sin 2 \rho \sin \delta \\
z_{44}=\cos \delta
\end{array}\right.
$$

Here $\rho$ is the direction of optical axis determined by the direction of packing of the birefringent fibril, $\delta=(2 \pi / \lambda) \Delta n d$ is the phase shift introduced between the orthogonal components of the amplitude of laser wave with the length $\lambda$ passing through the fibril with linear size of its geometrical section $d$ and birefringence index $\Delta n$.

Mueller matrix $\left(Z_{i k}\right)_{j}$ elements of the net of protein fibrils of partial BT layer $(j)$ are determined by the following algorithms: (i) for finite number $(N)$ of fibrils

$$
Z_{i k}=\sum_{u=1}^{N} \sum_{u=1}^{N} z_{i k}\left(\rho_{n}, \delta_{n}\right),
$$

(ii) for "infinite" $(N \rightarrow \infty)$ number of fibrils

$$
Z_{i k}=\int_{0}^{\pi} \int_{0}^{2 \pi} Q_{\rho} W_{\delta} z_{i k}(\rho, \delta) d \rho d \delta .
$$

Here $Q_{\rho}$ and $W_{\delta}$ are the distribution function of orientation $(\rho)$ and phase $(\delta)$ parameters of biological crystals network.

Mueller matrix of optically thick BT is determined by multiplication of partial matrix operators:

$$
\{Z\}=\{Z\}_{n}\{Z\}_{n-1} \cdots\{Z\}_{2}\{Z\}_{1} .
$$

To make it simpler (without decreasing the analysis depth) further we shall consider only a 2-layered BT:

$$
\{Z\}=\{Z\}_{2}\{Z\}_{1} \equiv\{Y\}\{X\} .
$$

In the expanded form the matrix operator elements (6) are written as follows:

$$
Z_{i k}\left(\rho_{x}, \rho_{y}, \delta_{x}, \delta_{y}\right)=\left\{\begin{array}{l}
Z_{22}=y_{22} x_{22}+y_{23} x_{32}+y_{24} x_{42}, \\
Z_{23}=y_{22} x_{23}+y_{23} x_{33}+y_{24} x_{43}, \\
Z_{32}=y_{32} x_{22}+y_{33} x_{32}+y_{34} x_{42}, \\
Z_{33}=y_{32} x_{23}+y_{33} x_{33}+y_{34} x_{43}, \\
Z_{34}=y_{32} x_{24}+y_{33} x_{34}+y_{34} x_{44}, \\
Z_{43}=y_{42} x_{23}+y_{43} x_{33}+y_{44} x_{43}, \\
Z_{24}=y_{22} x_{24}+y_{23} x_{34}+y_{24} x_{44}, \\
Z_{42}=y_{42} x_{22}+y_{43} x_{32}+y_{44} x_{42}, \\
Z_{44}=y_{42} x_{24}+y_{43} x_{34}+y_{44} x_{44} .
\end{array}\right.
$$

The analysis of relations (7) shows that optical properties of anisotropic component of 2-layered BT $\left(Z_{i k}\right)$ are described by complex superposition of orientation and phase parameters of its partial layers' biological crystals $\left(x_{i k}\left(\rho_{x}, \delta_{x}\right), y_{i k}\left(\rho_{y}, \delta_{y}\right)\right)$. The concrete form of such dependences for "phase" $z_{42,43,44}$ matrix elements [10] is illustrated by the following relations:

$$
\begin{aligned}
z_{24}= & \sin \delta_{y}\left[\cos 2 \rho_{x} \sin 2\left(\rho_{x}-\rho_{y}\right)\right. \\
& \left.+\cos \delta_{x} \sin 2 \rho_{x} \cos 2\left(\rho_{x}+\rho_{y}\right)\right] \\
+\sin \delta_{x} \cos \delta_{y} \sin 2 \rho_{x} ; & \\
z_{34}=\sin \delta_{x}\left[\sin 2 \rho_{y} \sin 2\left(\rho_{x}-\rho_{y}\right)\right. & \left.\quad+\cos \delta_{y} \cos 2 \rho_{y} \cos 2\left(\rho_{x}+\rho_{y}\right)\right] \\
& +\cos \delta_{x} \sin \delta_{y} \cos 2 \rho_{y} ; \\
z_{44}= & \cos \left(2 \rho_{x}-2 \rho_{y}\right) \sin \delta_{x} \sin \delta_{y}+\cos \delta_{x} \cos \delta_{y} .
\end{aligned}
$$




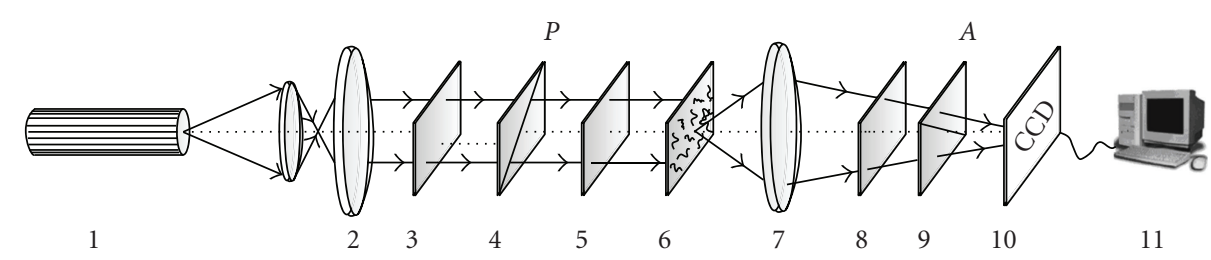

FIGURE 2: Optical scheme of polarimeter, where 1: He-Ne laser; 2: collimator; 3: stationary quarter-wave plate; 5, 8: mechanically movable quarter-wave plates; 4, 9: polarizer and analyzer correspondingly; 6: object of investigation; 7: microobjective; 10: CCD camera; 11: PC.

The analysis of relations ( 8 ) shows that a strict solution of the inverse problem $\left\{\rho_{x}=q\left(z_{i k}, \rho_{y}, \delta_{y}\right) ; \delta_{x}=g\left(z_{i k}, \rho_{y}, \delta_{y}\right)\right\}$, revealing the changes in the structure of biological crystals network of one of the layers on the basis of data about matrix elements $z_{i k}$ and $y_{i k}$, is both mathematically incorrect and physically ambiguous.

Thus, it is important to elaborate approximated statistical methods of experimental solution of such diagnostic task.

Generally speaking, the increase of optical thickness of biological layer results in multiple scattering regime in its volume. Such process appeared in formation of integral depolarization of laser field and results in averaging within angular aperture of photodetector of azimuth and ellipticity random values of fully polarized isolated speckles [8]. In this case the direct interconnection between the scattered field polarization parameters and the orientation and phase structure of biological crystals will be lost. From the other hand, for such integrally depolarized fields the Mueller matrix approach remains adequate [1]. Therefore, the search of interconnections "object-laser field" on the level of indirect, statistic parameters, which characterizes their structure is topical.

\section{Scheme of Experimental Changes of Coordinate Distributions of Biological Tissue Mueller Matrix Elements}

The histological sections of different geometrical (optical) thickness were used as the objects of investigation. The technique of obtaining of such objects is convenient: biological tissue is freezing to nitrogen temperature with the following obtaining, by means of medical microtome, the histological sections (from $10 \mu \mathrm{m}$ to $100 \mu \mathrm{m}$ ). Such a technique allows preserving the optical properties of biological tissues over a period of 24 hours.

Conventional optical scheme of polarimeter for measuring $2 \mathrm{D}$ distributions of the BT Mueller matrix elements is presented in Figure 2 [1].

Histological sections of BT were illuminated by a parallel beam of He-Ne laser $(\lambda=0.6328 \mu \mathrm{m}, W=5.0 \mu \mathrm{W})$ with the radius $r=1 \mathrm{~mm}$. Polarization illuminator consists of quarter-wave plates 3,5 and polarizer 4, providing the formation of laser beam with random azimuth $0^{\circ} \leq \alpha_{0} \leq$ $180^{\circ}$ or ellipticity $0^{\circ} \leq \beta_{0} \leq 90^{\circ}$ of polarization. Polarization images of BT by means of microobjective 7 were projected into the plane of sensitized plate $(m \times n=800 \times 600$ pixels $)$ if with CCD-camera 10. The analysis of BT images was carried out by means of polarizer 9 and quarter-wave plate 8 . As a result, the Stokes vector parameters for every pixel of the BT image $\left\{S_{i=1,2,3,4}\right\}$ were determined and the set of elements of Mueller matrix was calculated according to algorithm [10]:

$$
\begin{aligned}
& Z_{i 1}=0,5\left[S_{i}^{(1)}+S_{i}^{(1)}\right], \\
& Z_{i 2}=0,5\left[S_{i}^{(1)}-S_{i}^{(1)}\right], \\
& Z_{i 3}=S_{l}^{(3)}-Z_{i 1}, \\
& Z_{i 4}=S_{l}^{(4)}-Z_{i 1}, \quad i=1,2,3,4 .
\end{aligned}
$$

Indices $1-4$ correspond to the following polarization states of the beam probing the BT layer: $1-0^{\circ} ; 2-90^{\circ} ; 3-+45^{\circ} ; 4-\otimes$ (right-hand circulation).

The technique of measuring the ensemble ( $j$ - the number of probing areas) of 2D matrix elements $Z_{i k}{ }^{(j)}(m \times n)$ of BT consisted in the following sequence of actions:

(i) the plane of BT histological sections $(\approx 10 \mathrm{~mm} \times$ $20 \mathrm{~mm}$ ) BT was scanned line by line by the laser beam with the radius $r=1 \mathrm{~mm}$ with the step of linear displacement $\Delta r=2 \mathrm{~mm}$;

(ii) within each of 50 areas $(j=1,2, \ldots, 50)$ of illumination of the BT layer plane according to the algorithm (9) the local array $(m \times n=800 \times 600)$ of values of Mueller matrix elements $Z_{i k}{ }^{(j)}(m \times n)$ was determined:

$$
Z_{i k}^{(j)}(m \times n)=\left(\begin{array}{lll}
Z_{i k}^{11} & \cdots & Z_{i k}^{1 m} \\
\cdots & \cdots & \cdots \\
Z_{i k}^{n 1} & \cdots & Z_{i k}^{n m}
\end{array}\right)_{j=1,2, \ldots, 50}
$$

(iii) for every local array $Z_{i k}^{(j)}(m \times n)$ statistic moments of the 3rd-4th $M^{(s=3,4)}$ orders were calculated;

(iv) the histograms $N\left(M_{j=1,2, \ldots, 50}^{(s=3 ; 4)}\right)$ of the values of statistical moments of higher orders within the whole plane of the investigated BT histological sections were determined. 


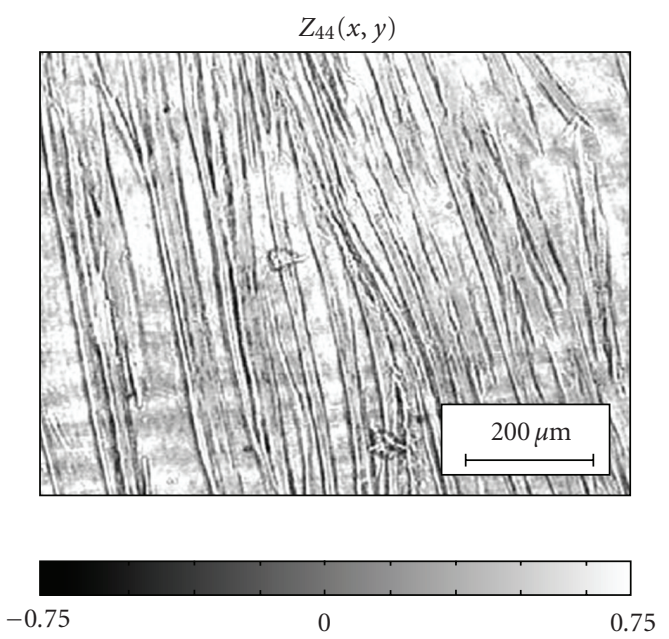

(a)

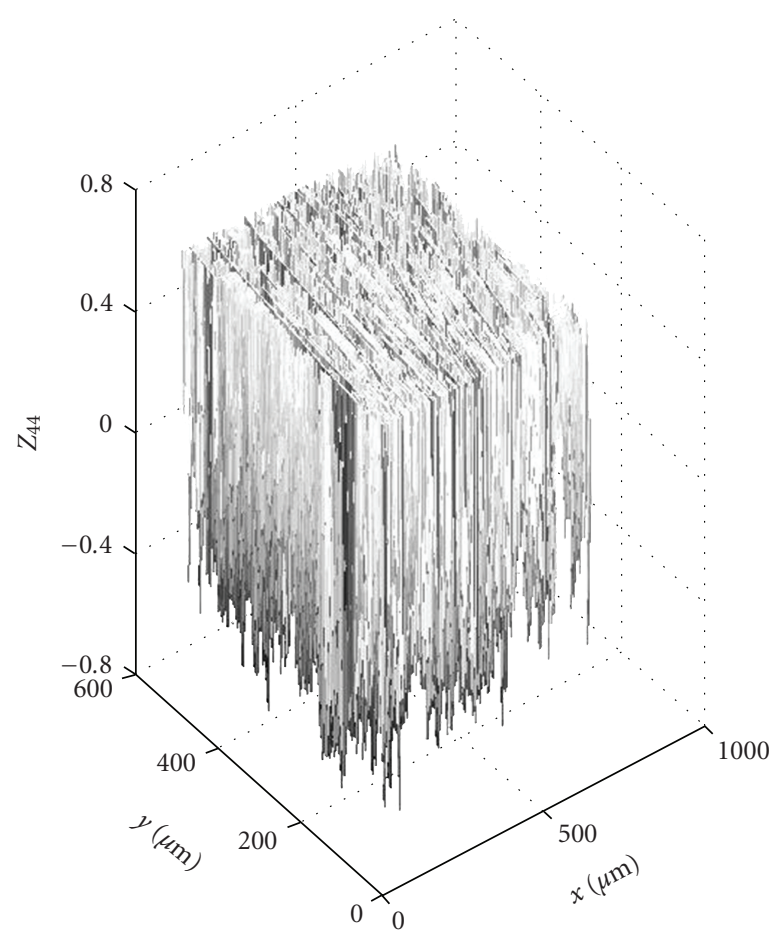

(b)

FIgURE 3: Coordinate and 3D distribution of the values of phase matrix element $Z_{44}(m, n)$ of histological section of optically thin $\left(\tau_{1}=0,08\right)$ layer of the healthy rat's muscle tissue.

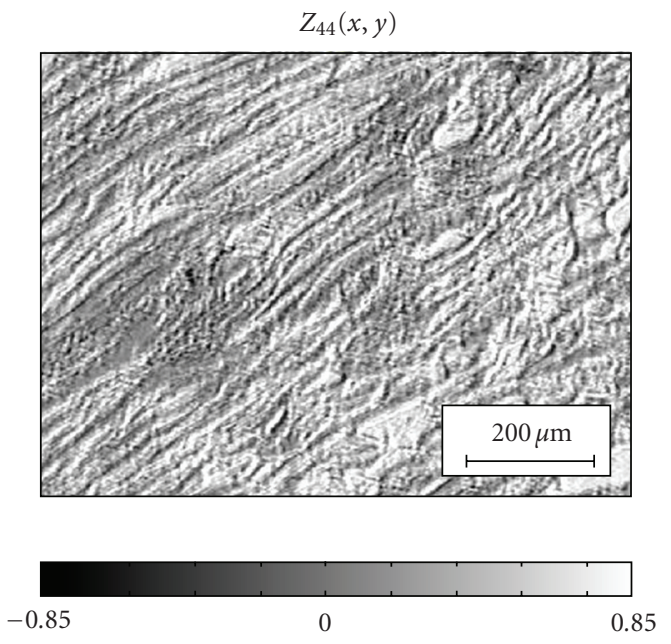

(a)

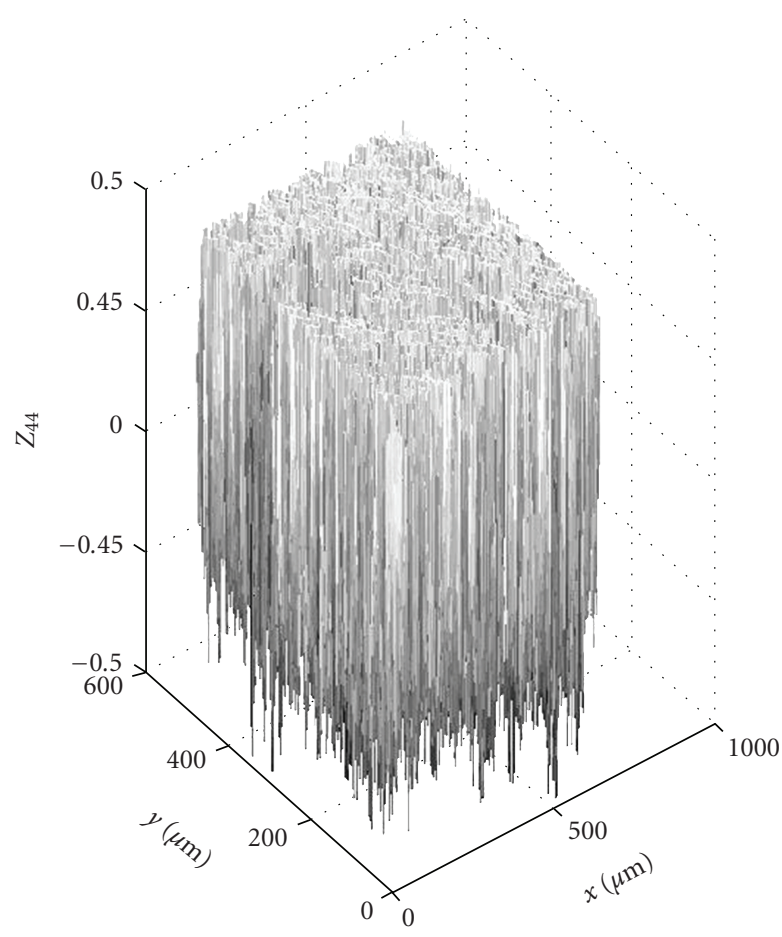

(b)

FIGURE 4: Coordinate and 3D distribution of the values of phase matrix element $Z_{44}(m, n)$ of histological section of optically thin $\left(\tau_{1}=0,08\right)$ layer of the inflamed rat's muscle tissue. 


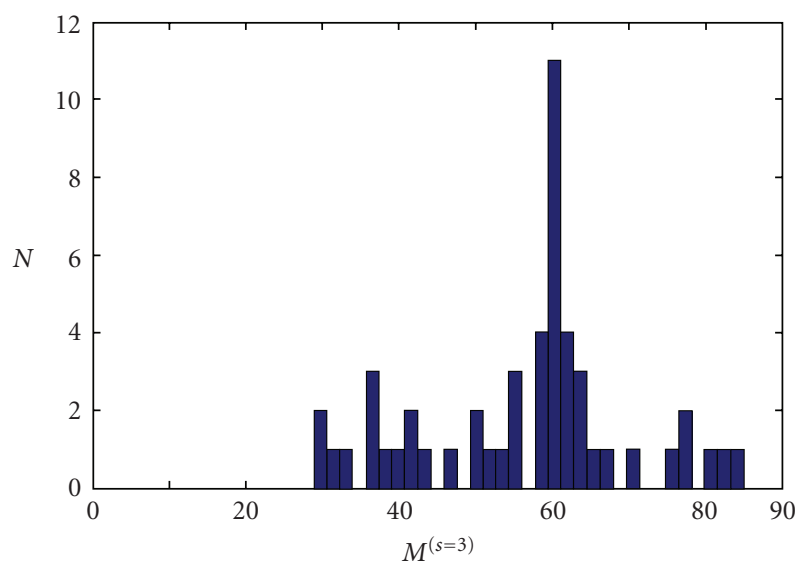

(a)

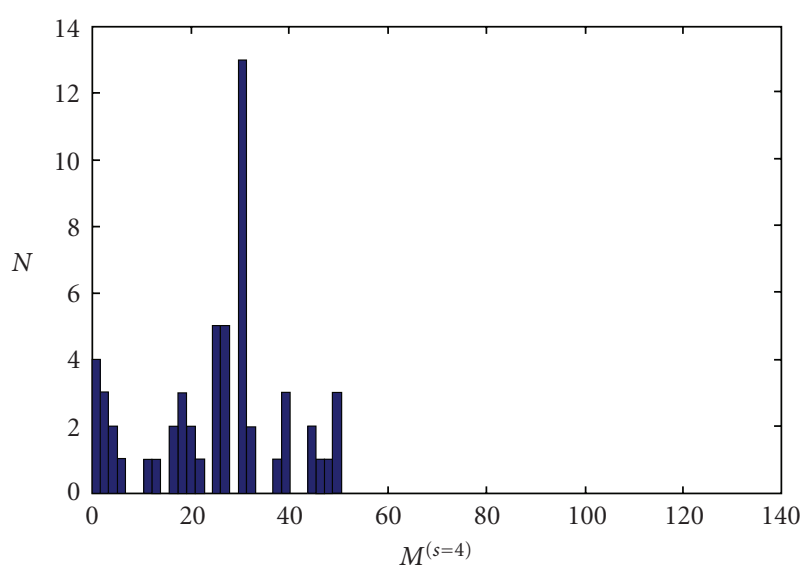

(c)

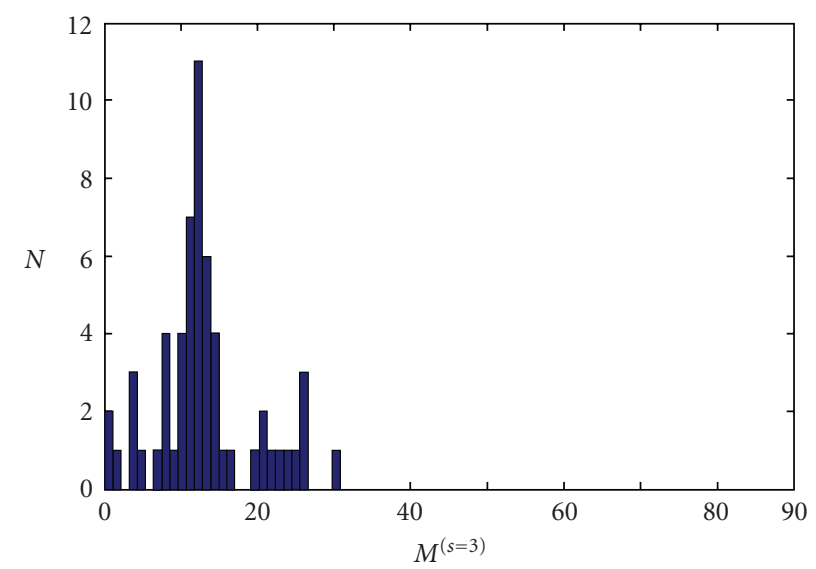

(b)

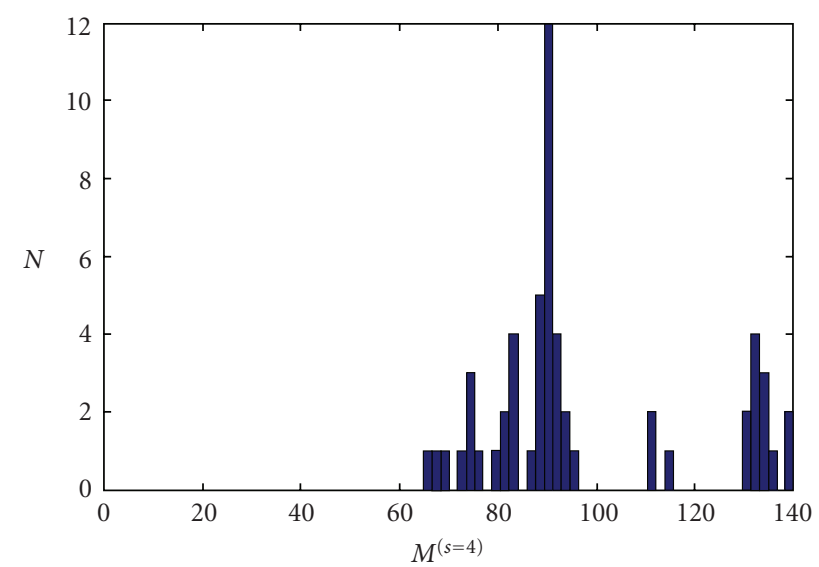

(d)

FIgURE 5: Histograms of the set of skewness $M^{(s=3)}$ values $(\mathrm{a}, \mathrm{b})$ and kurtosis $M^{(s=4)}$ (c, d) of coordinate distribution of the element $Z^{(j=1-50)}{ }_{44}(m, n)$ of histological section of optically thin $\left(\tau_{1}=0,08\right)$ layer of the healthy $(\mathrm{a}, \mathrm{c})$ and inflamed (b, d) rat's skeletal tissue.

\section{Investigation of Skewness and Kurtosis Distributions of 2D Phase Element of Mueller Matrix of the Samples of Skeletal Muscle Tissue}

The following histological sections of different types of rat's tissues were investigated-connection tissue, muscular tissue, epithelial tissue, and nervous tissue. All these tissues have common optical peculiarity-the presence of birefringent network of protein fibrils [1]. Therefore, in this work, without decrease of analysis completeness, we have concentrated on investigation of optical anisotropy of rat's skeletal muscle tissue birefringent fibrils.

Distributions $N\left(M^{(s=3,4)}\right)$ of the values of skewness $M^{(s=3)}$ and kurtosis $M^{(s=4)}$ of $2 \mathrm{D}$ phase $\left(Z_{44}\right)$ element of Mueller matrix of rat's skeletal muscle tissue were investigated. Histological sections of various optical thickness $\left(\tau_{1}=0,08\right.$ and $\left.\tau_{2}=1,47\right)$ and physiological state were the object of investigation. Such choice of optical thickness of histological sections $\left(d_{1}=15 \mu \mathrm{m} ; d_{2}=50 \mu \mathrm{m}\right)$ allows us to investigate the influence of a single $\left(\tau_{1}=0,08\right)$ and multiple $\left(\tau_{2}=1,47\right)$ scattering on coordinate structure of Mueller matrix elements.
The choice of matrix element $Z_{44}$ as an analytical parameter is explained by the fact that it is the most sensitive to the changes of birefringence of protein fibrils nets, connected with their pathological changes $[1,11]$. The choice of a skeletal muscle tissue of a rat as the object of investigation is connected with the possibility of direct experimental formation of septic inflammation and monitoring the control of their optical manifestations under the condition of scattering of laser beam of various multiplication factor.

The series of Figures 3 and 4 presents the distributions of the element $Z_{44}(m, n)$ of optically thin histological sections of healthy (Figure 3 ) and inflamed (Figure 4) muscle tissue.

The following can be seen from the data obtained:

(i) 2D distributions of matrix elements $Z_{44}(m, n)$ of both types of tissues are coordinately heterogeneous;

(ii) for the sample of healthy muscle tissue (Figure 3 ) the lesser range of values change (from -0.7 to 0.7 ) of the matrix element $Z_{44}(m, n)$ is typical in comparison with the changes (from -0.85 to 0.85 ) of the analogous element of Mueller matrix of the inflamed tissue (Figure 4). 


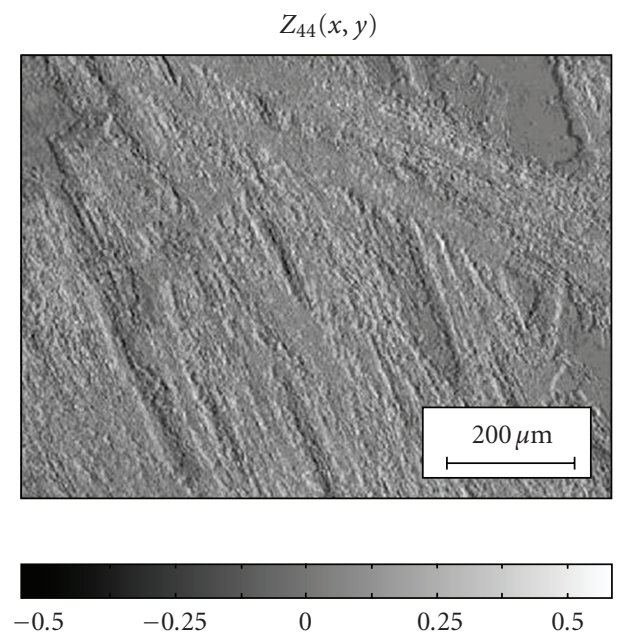

(a)

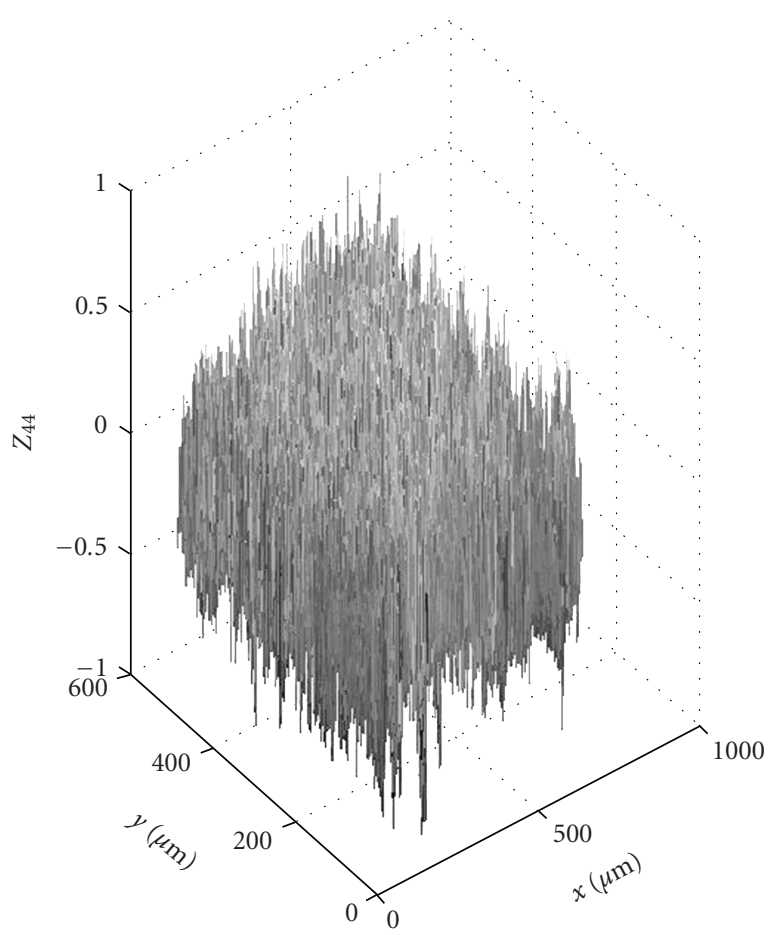

(b)

FIGURE 6: Coordinate and 3D distribution of the values of phase matrix element $Z_{44}(m, n)$ of histological section of optically thick $\left(\tau_{1}=1,47\right)$ layer of the healthy rat's muscle tissue.

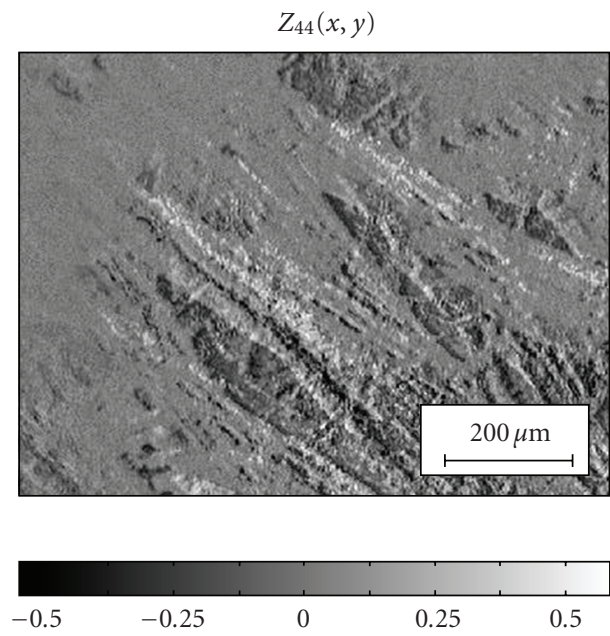

(a)

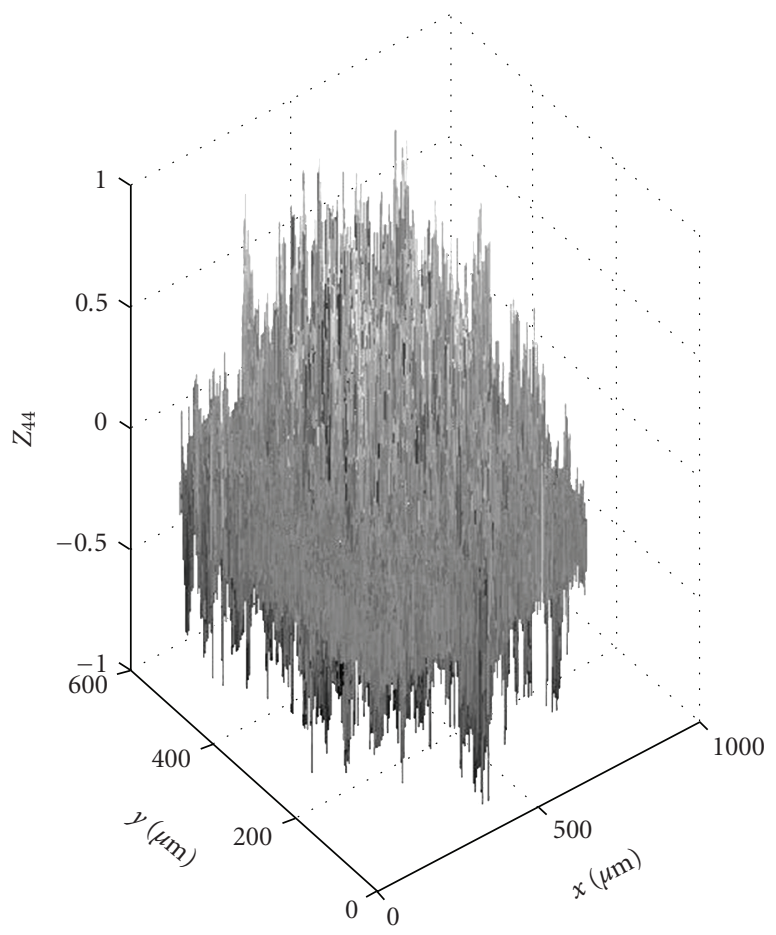

(b)

FIGURE 7: Coordinate and 3D distribution of the values of phase matrix element $Z_{44}(m, n)$ of histological section of optically thick $\left(\tau_{1}=1,47\right)$ layer of the inflamed rat's muscle tissue. 


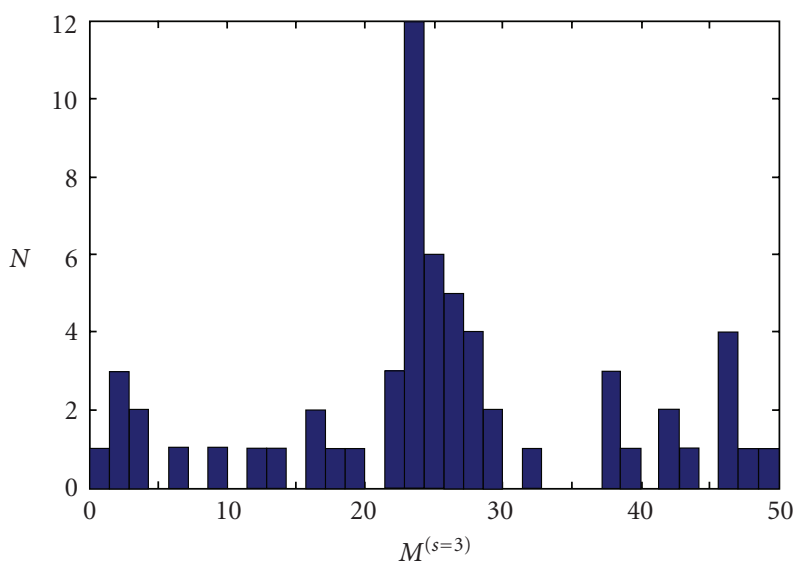

(a)

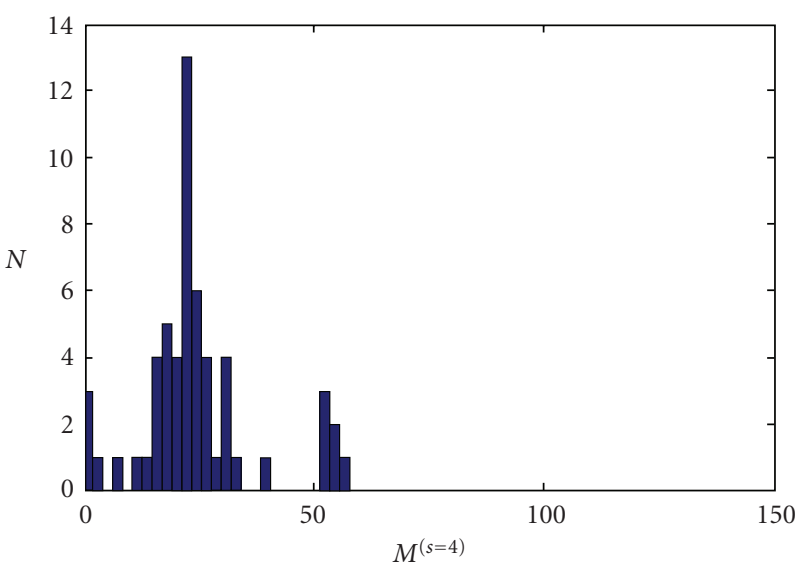

(c)

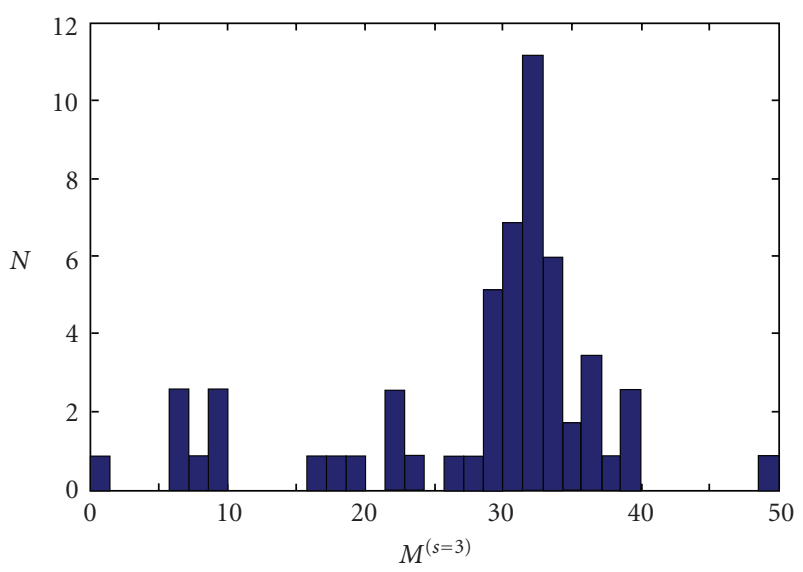

(b)

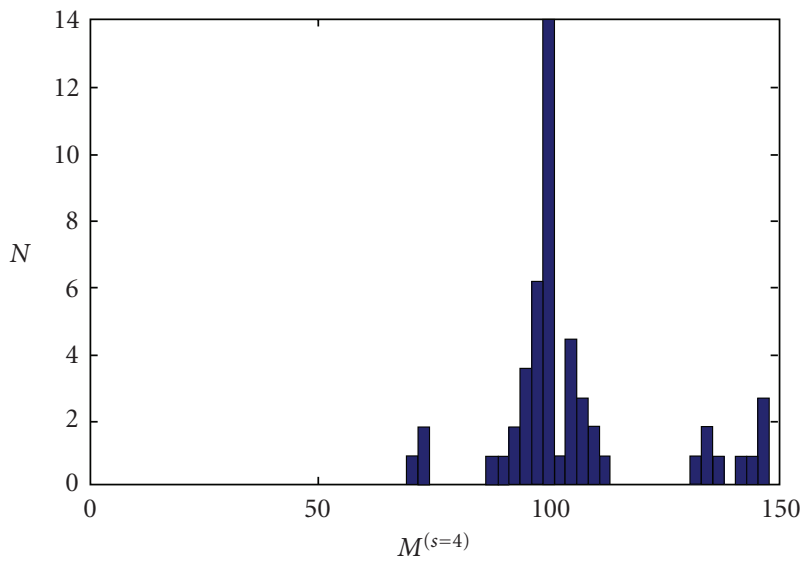

(d)

FIgURE 8: Histograms of the set of skewness $M^{(s=3)}$ values $(\mathrm{a}, \mathrm{b})$ and kurtosis $M^{(s=4)}$ (c, d) of coordinate distribution of the element $Z^{(j=1-50)}{ }_{44}(m, n)$ of histological section of optically thick $\left(\tau_{2}=1,17\right)$ layer of the healthy $(\mathrm{a}, \mathrm{c})$ and inflamed (b, d) rat's skeletal tissue.

The structure of $2 \mathrm{D}$ distributions of elements $Z_{44}(m, n)$ can be connected with the following peculiarities of birefringent architectonics of the skeletal tissue samples. Firstly, while a laser wave (with the wave-length $\lambda$ ) propagates through the network of optically anisotropic (birefringence index $\Delta n=1,5 \times 10^{-3}$ [7]) myosin fibrils, a wide range of values of phase shifts $\delta_{k}$, proportional to their geometrical sizes $d_{k}$, is formed

$$
\delta_{k} \sim\left(\frac{2 \pi \Delta n}{\lambda}\right) \times\left\{\begin{array}{l}
d_{1} \\
\vdots \\
d_{k}
\end{array}\right.
$$

Thus, phase elements $Z_{44}(m, n)$ distributions are coordinately heterogeneous and dependent on the peculiarities of morphological structure of extracellular matrix of muscle tissue samples.

Secondly, the inflammation process is accompanied by the increase of birefringence $\Delta n$ due to formation of myosin fibrils edema [7]. Optically, it causes the increase of the range of phase shifts $\delta_{k}$ changes and the growth of fluctuations of the element $Z_{44}(m, n)$ connected with it (Figure 4).
The histograms of distribution $N\left(M^{(s=3,4)}\right)$ of statistic moments of higher-orders $M^{(s=3,4)}$ of coordinate distributions $Z^{(j=1 \div 50)} 44(m, n)$ of investigated samples of optically thin histological sections are presented in Figure 5.

The analysis of experimentally measured histograms $N\left(M^{(s=3,4)}\right)$ proved the following:

(i) the change ranges of the values of skewness $M^{(s=3)}$ and kurtosis $M^{(s=4)}$ of coordinate distributions $Z^{(j=1-50)} 44(m, n)$, measured for the healthy $(\mathrm{a}, \mathrm{c})$ and inflamed (b,d) muscle tissues, do not actually coincide;

(ii) extreme values of skewness in the distribution $N\left(M^{(s=3)}\right)$ for phase element $Z_{44}$ of the healthy tissue are by $4-6$ times higher than analogous values of the given statistic moment $M^{(s=3)}$ for the inflamed muscle tissue;

(iii) extreme values of kurtosis in the distribution $N\left(M^{(s=4)}\right)$ for the inflamed tissue are by $2-3$ times higher than the values of the statistic moment $M^{(s=4)}$ for the healthy skeletal tissue. 
Thus, it can be stated that for single differentiation of physiological state of optically thin layers of muscle tissue it is enough to measure the $2 \mathrm{D}$ phase matrix element $Z_{44}(m, n)$ in one domain $\left(\pi r^{2}\right)$ of irradiation by the laser beam and to calculate the skewness and kurtosis of its values distribution.

The series of Figures 6 and 7 presents the results of experimental measurements of phase elements $Z_{44}(m, n)$ of Mueller matrix of optically thick $\left(\tau_{2}=1,47\right)$ of the rat's muscle tissue layers.

It can be seen from the data obtained the following:

(i) for the samples of the healthy and inflamed muscle tissue the same range of values change (from $-0,55$ to $0,55)$ is typical in the distribution of matrix elements $Z_{44}(m, n)$;

(ii) comparative visual analysis of coordinate distributions of matrix elements $Z_{44}(m, n)$ of histological sections of skeletal tissue of both types did not show any sufficient difference between them.

Similarity of the distribution structure $Z_{44}(m, n)$ of both types of rat's skeletal tissue samples can be explained by multiple light-scattering. As a result of every local (ith) act of interaction between laser radiation and separate fibril random value of phase shift $\delta^{(i)}$ is formed, which is multiplied $\left(\delta^{*}=\sum_{i=1}^{R} \delta_{i}\right)$ in the process of propagation in the BT depth reaching equiprobable random values from 0 to $2 k \pi, k=1,2,3 \ldots$.

Figure 8 shows histograms $N\left(M^{(s=3,4)}\right)$ of the set of values of statistic moments of skewness $M^{(s=3)}$ and kurtosis $M^{(s=4)}$ of distributions of the matrix element $Z^{(j=1-50)}{ }_{44}(m, n)$ of optically thick samples of muscle tissue.

The analysis of experimental data showed the following:

(i) the skewness $M^{(s=3)}$ values distribution of 2D phase $Z^{(j=1-50)} 44(m, n)$, and changes within the whole plane of samples of the layer of the healthy (a) and inflamed (b) muscle tissue practically coincide and cannot be used as an objective criterion for their optical properties differentiation;

(ii) histograms $N\left(M^{(s=4)}\right)$ of the values of the statistic moment $M^{(s=4)}$ of elements $Z^{(j=1-50)} 44(m, n)$ distribution possess individual structure that depends on physiological state of muscle tissue;

(iii) extreme values of kurtosis $M^{(s=4)}$ in the histogram $N\left(M^{(s=4)}\right)$ of the healthy tissue (c) are by 4-5 times less than analogous values of the given statistic moment of the inflamed rat's skeletal tissue (d).

\section{Conclusion}

Thus for realization of Mueller-matrix differentiation of optically thick BT layers at early stages of changing their physiological state the measurement of distributions of the kurtosis $N\left(M^{(s=4)}\right)$ of the ensemble of phase elements $Z^{(j=1 \div 50)} 44(m, n)$ is objective.
The Mueller-matrix approach in diagnostics and differentiation of the biological crystals networks appears to be effective for optically thick histological sections layers of human tissues (skin derma, tissues of women reproductive sphere, parenchymatous tissues) also.

\section{References}

[1] A. G. Ushenko and V. P. Pishak, Coherent-Domain Optical Methods. Biomedical Diagnostics, Environmental and Material Science, Kluwer Academic Publishers, Dordrecht, The Netherlands, 2004.

[2] J. F. de Boer and T. E. Milner, "Review of polarization sensitive optical coherence tomography and Stokes vector determination," Journal of Biomedical Optics, vol. 7, no. 3, pp. 359-371, 2002.

[3] J. F. de Boer, T. E. Milner, and J. S. Nelson, Trends in Optics and Photonics (TOPS): Advances in Optical Imaging and Photon Migration, OSA, Washington, DC, USA, 1998.

[4] M. J. Everett, K. Schoenenberger, B. W. Colston Jr., and L. B. Da Silva, "Birefringence characterization of biological tissue by use of optical coherence tomography," Optics Letters, vol. 23, no. 3, pp. 228-230, 1998.

[5] S. Jiao, W. Yu, G. Stoica, and L. V. Wang, "Optical-fiber-based Mueller optical coherence tomography," Optics Letters, vol. 28, no. 14, pp. 1206-1208, 2003.

[6] O. V. Angelsky, G. V. Demianovsky, A. G. Ushenko, D. N. Burkovets, and Yu. A. Ushenko, "Wavelet analysis of two-dimensional birefringence images of architectonics in biotissues for diagnosing pathological changes," Journal of Biomedical Optics, vol. 9, no. 4, pp. 679-690, 2004.

[7] O. V. Angelsky, Yu. Ya. Tomka, A. G. Ushenko, Ye. G. Ushenko, S. B. Yermolenko, and Yu. A. Ushenko, "2-D tomography of biotissues images in pre-clinic diagnostics of their pre-cancer states," in Advanced Topics in Optoelectronics, Microelectronics, and Nanotechnologies II, Proceedings of the SPIE, 2004.

[8] O. V. Angelsky, A. G. Ushenko, D. N. Burkovets, and Yu. A. Ushenko, "Polarization visualization and selection of biotissue image two-layer scattering medium," Journal of Biomedical Optics, vol. 10, no. 1, Article ID 014010, 2005.

[9] O. V. Angelsky, Yu. Ya. Tomka, A. G. Ushenko, Ye. G. Ushenko, and Yu. A. Ushenko, "Investigation of 2D Mueller matrix structure of biological tissues for pre-clinical diagnostics of their pathological states," Journal of Physics D, vol. 38, no. 23, pp. 4227-4235, 2005.

[10] Yu. A. Ushenko, "Statistical structure of polarizationinhomogeneous images of biotissues with different morphological structures," Ukrainian Journal of Physical Optics, vol. 6, pp. 63-70, 2005.

[11] O. V. Angelsky, A. G. Ushenko, and Yu. A. Ushenko, "Polarization reconstruction of orientation structure of biological tissues birefringent architectonic nets by using their Muellermatrix speckle-images," Journal of Holography and Speckle, vol. 2, pp. 72-79, 2005.

[12] O. V. Angelsky, P. P. Maksimyak, V. V. Ryukhtin, and S. G. Hanson, "New feasibilities for characterizing rough surfaces by optical-correlation techniques," Applied Optics, vol. 40, no. 31, pp. 5693-5707, 2001.

[13] O. V. Angelsky, P. P. Maksimyak, and T. O. Perun, "Optical correlation method for measuring spatial complexity in optical fields," Optics Letters, vol. 18, no. 2, pp. 90-92, 1993. 

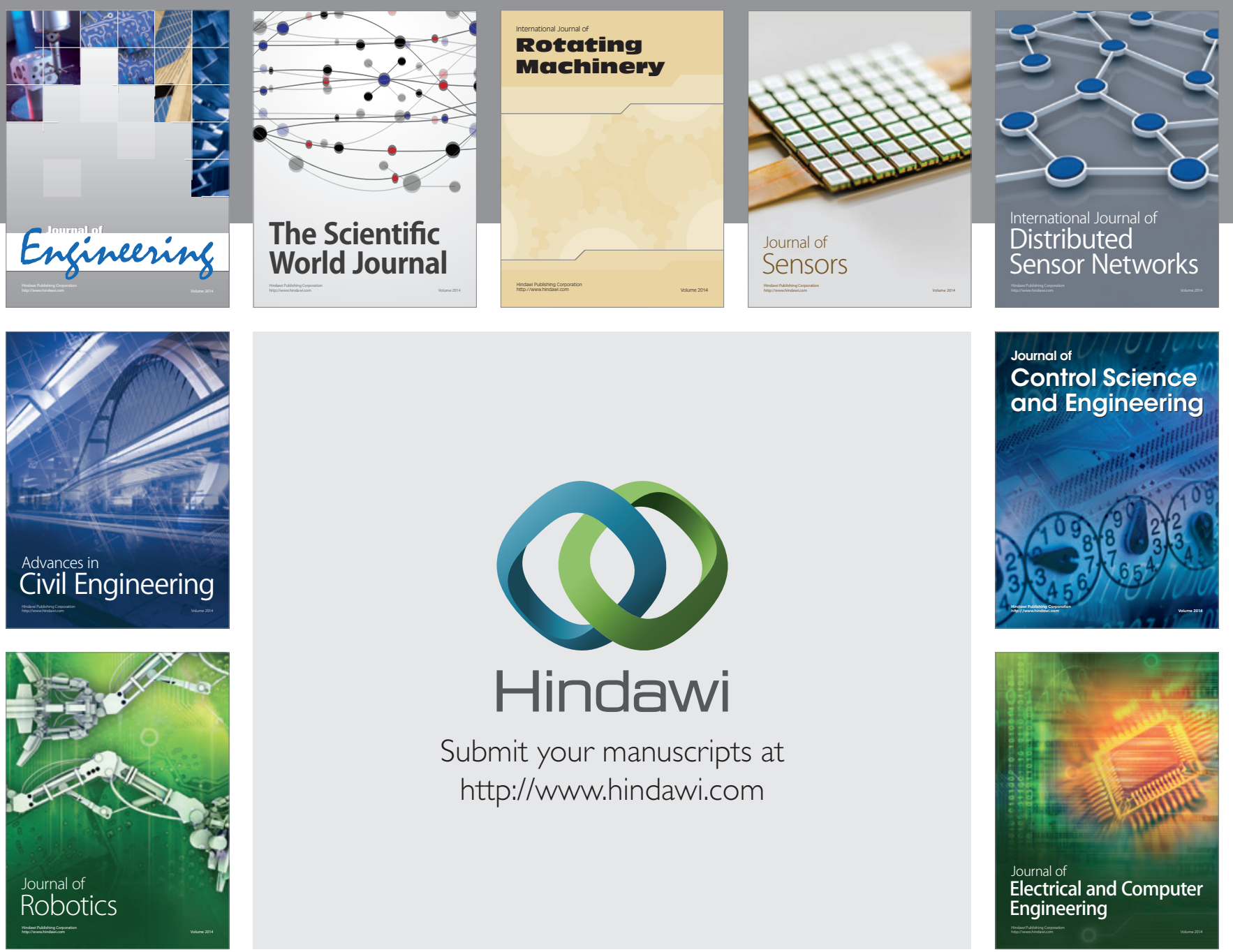

Submit your manuscripts at

http://www.hindawi.com
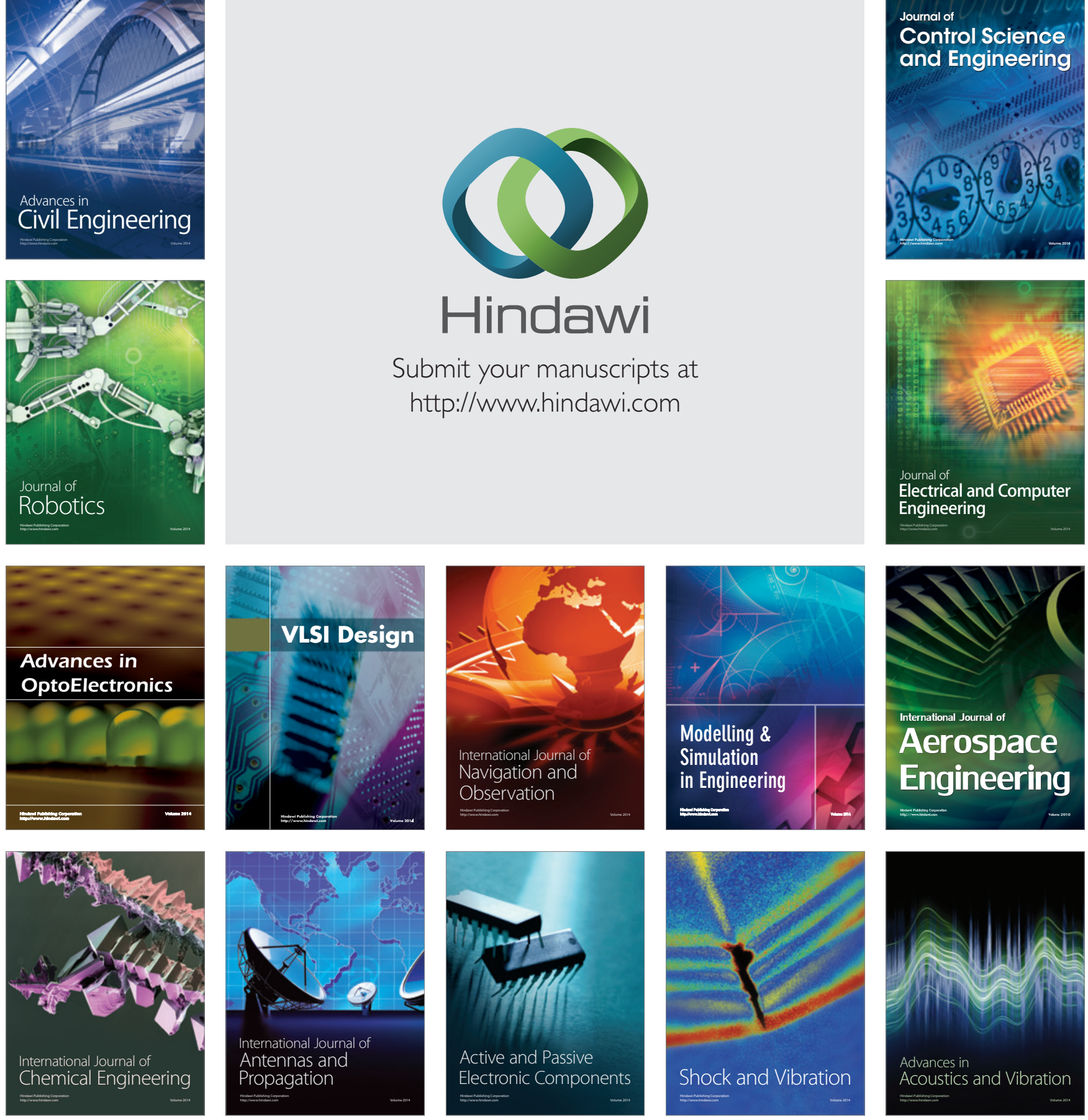